ARTIGO

DE REVISÃO

\title{
Consumo de carboidratos e lipídios no desempenho em exercícios de ultra-resistência
}

\author{
Antonio Marcio Domingues Ferreira ${ }^{1}$, Beatriz Gonçalves Ribeiro ${ }^{2}$ e Eliane de Abreu Soares ${ }^{2}$
}

\section{RESUMO}

A nutrição é uma importante ferramenta dentro da prática desportiva. Dentre as modalidades esportivas, a nutrição exerce uma grande influência nos chamados "esportes de desafio", que são as provas de ultra-resistência ou de longa duração. O custo energético de uma prova de ultraresistência pode variar de 5.000 a $18.000 \mathrm{kcal}$ por dia. É amplamente aceito que o consumo de carboidratos antes e durante exercícios prolongados irá retardar o aparecimento da fadiga, poupando o glicogênio hepático e muscular e fornecendo glicose diretamente para os músculos em atividade. Recomenda-se que a dieta de atletas de ultra-resistência possua $70 \%$ ou mais, ou de 7 a 10 gramas por quilo de peso corporal de carboidratos. Porém, apesar da melhora apresentada com a nutrição bem planejada, alguns pesquisadores procuram desenvolver novas intervenções nutricionais, visando a melhora do rendimento, que continuam a ser estudadas, como a suplementação com lipídios, através do consumo de triglicerídeos de cadeia média (TCM) ou de dietas ricas em lipídios nos dias que antecedem a competição. Sendo assim, esta revisão possui como objetivo elucidar como os carboidratos e os lipídios podem influenciar o desempenho nos exercícios de ultra-resistência.

Palavras-chave: Nutrição. Desempenho. Exercício de ultra-resistência. Carboidratos. Lipídios.

1. Escola de Educação Física e Desportos - Universidade Federal do Rio de Janeiro, Centro de Capacitação Física do Exército, Rio de Janeiro, RJ.

2. Instituto de Nutrição - Universidade Federal do Rio de Janeiro, Rio de Janeiro, RJ.

Recebido em: 27/11/2000.

Aceito em: 28/3/2001.

Endereço para correspondência:

Antonio Marcio Domingues Ferreira

Rua Pontes Corrêa, 147, casa 4, Andaraí

20510-050 - Rio de Janeiro, RJ

Tel.: (21) 268-1262

E-mail: amdf28@ @erra.com.br

\section{ABSTRACT \\ Consumption of carbohydrates and lipids in ultra-endur- ance exercise performance}

Nutrition is an important tool in the sports universe. Among all sport modalities, nutrition has great influence on the "challenge sports", also known as ultra-endurance competitions. The energetic cost of an ultra-endurance event can vary from 5,000 to $18,000 \mathrm{kcal}$ a day. The diet recommendation for ultra-endurance athletes is $70 \%$ or more, or 7 to 10 grams per kilogram of body weight, of carbohydrates. It is fully accepted that the ingestion of carbohydrates before and during prolonged exercises will delay fatigue, saving the hepatic and muscular glycogen and providing glucose directly to the active muscles. However, although well-planned nutrition shows improvement, some researchers continue to develop new ways of improving performance, like lipid supplementation through the ingestion of medium-chain triglycerides during exercise or a high-fat diet during the days before competition. Therefore, the aim of this revision is to elucidate how carbohydrates and lipids can influence performance in ultra-endurance exercises.

Key words: Nutrition. Performance. Ultra-endurance exercise. Carbohydrates. Lipids.

\section{INTRODUÇÃO}

A participação em competições desportivas pela população em geral vem aumentando largamente. Dentre essas competições, as que despertam um maior fascínio são as chamadas provas de ultra-resistência ou de longa duração. Segundo Moreira (1996) ${ }^{1}$, a cada ano verifica-se um número maior de inscrições nos, por ele chamados, "esportes de desafio", como por exemplo as Super e Ultra-Maratonas (a partir de $84 \mathrm{~km}$ ), o Ironman Triathlon $(3,8 \mathrm{~km}$ de natação, $180 \mathrm{~km}$ de ciclismo e $42 \mathrm{~km}$ de corrida), provas que duram mais de 24 horas, como o Ultraman Triathlon $(10 \mathrm{~km}$ de natação, $421 \mathrm{~km}$ de ciclismo e $84 \mathrm{~km}$ de corrida) e provas de ciclismo que chegam a durar até 30 dias (Tour de 
France, Vuelta Ciclista a España, Giro de Italia e Race Across America).

Esse crescente interesse na participação em provas de longa duração vem ocasionando também um maior interesse na melhora que a nutrição pode promover sobre o desempenho ${ }^{2}$.

Segundo McMurray e Anderson (1996) ${ }^{3}$, a nutrição é um dos fatores que pode favorecer o desempenho atlético, já que, quando bem orientada, pode reduzir a fadiga, permitindo que $o$ atleta treine por mais tempo ou que se recupere melhor entre os treinos; reduzir as lesões ou ajudar na recuperação das mesmas; aumentar os depósitos de energia para a competição; e finalmente por ajudar a saúde geral do atleta.

Em um estudo realizado com ciclistas durante uma competição, Garcia-Rovés et al. (1998) ${ }^{4}$ observaram que os atletas consumiram uma quantidade acima do recomendado de lipídios, sendo esses provenientes de biscoitos e doces, um baixo volume de líquidos e uma quantidade de carboidratos abaixo do recomendado, concluindo os autores que a educação nutricional dos atletas, principalmente quanto ao consumo de líquidos, é a melhor maneira de se proceder para que estes possam render o máximo possível. Costill e Wilmore $(1999)^{5}$ acrescentam que atletas de ultraresistência podem ter problemas em equilibrar o consumo e a demanda energética, o que leva muitos deles a se preocuparem mais com a quantidade do que com a qualidade do que consomem. Ainda, segundo esses autores, muitos atletas procuram por um "alimento mágico" que irá promover um rendimento vencedor. No entanto, a maioria desses produtos e dietas são baseados em testemunhos pessoais, propagandas enganosas, estudos malconduzidos e na má interpretação de pesquisas nutricionais. Por causa disso, treinadores, nutricionistas, atletas e desportistas em geral devem estar sempre atualizados sobre os recursos nutricionais, como e quando utilizá-los.

Portanto, o objetivo desta revisão é elucidar a influência dos carboidratos e dos lipídios no desempenho em exercícios de ultra-resistência.

\section{GASTO ENERGÉTICO E CONSUMO ALIMENTAR}

A maior preocupação dos atletas de ultra-resistência é conseguir manter um ritmo considerado ideal durante toda a prova. Para isso, além de um treinamento físico bem direcionado, o atleta precisa consumir uma adequada quantidade de energia para sustentar as demandas do esforço físico realizado.

O substrato energético utilizado pelo organismo vai depender, segundo Basset e Nagle (1996) ${ }^{6}$ e Stroud (1998) ${ }^{7}$, da natureza, intensidade e duração do exercício; do consu- mo alimentar; do ambiente em que é realizado; e de uma variedade de fatores individuais como idade, sexo, peso e composição corporal, tipo de fibra muscular predominante, estado de treino e habilidade técnica.

As principais fontes de energia para os exercícios prolongados são os carboidratos e as gorduras ${ }^{8}$. No entanto, vem sendo demonstrado que o esforço físico prolongado e a depleção do glicogênio aumentam a parcela de contribuição protéica à demanda energética do exercício ${ }^{6,9}$.

O custo energético de uma prova de ultra-resistência está diretamente ligado à distância e ao ritmo da prova, da habilidade técnica do atleta e da sua massa corporal ${ }^{1,2,8,10}$. Dentre as provas de longa duração, as que requerem um maior gasto energético são as provas de corrida, visto que $o$ atleta precisa sustentar o peso do corpo, estando este fato diretamente ligado à massa corporal ${ }^{8,10}$, e as provas com um tempo de duração mais elevado.

Segundo Miller (1996) ${ }^{2}$, o gasto energético durante uma competição varia de $5.000 \mathrm{kcal}$ (triathlon com $2 \mathrm{~km}$ de natação, $90 \mathrm{~km}$ de ciclismo e $21 \mathrm{~km}$ de corrida) até $18.000 \mathrm{kcal}$ (corrida com 24 horas de duração). Stroud $(1998)^{7}$ cita um gasto energético de $6.430 \mathrm{kcal}( \pm 1.190)$ por dia, durante 7 dias, numa prova de $240 \mathrm{~km}$ através do Deserto do Saara. Já McArdle et al. (1999) ${ }^{11}$ relatam que a média do gasto energético durante o Tour de France é de 6.500kcal/dia, podendo chegar a $9.000 \mathrm{kcal} /$ dia nos estágios de montanha, e o gasto energético de uma Ultra-Maratona de $1.000 \mathrm{~km}$, durante 5 dias, é em média, de $59.079 \mathrm{kcal}$, com gasto diário variando entre 8.600 e $13.770 \mathrm{kcal}$.

Em virtude do grande gasto energético durante as competições e rotinas de treinamento, os atletas de ultra-resistência devem consumir $70 \%$ ou mais de carboidratos na $\operatorname{dieta}^{1,10,12-14}$, principalmente na semana anterior à competição. Atualmente, é preferível recomendar o consumo de carboidratos em gramas por quilo de peso corporal, sendo que a quantidade estimada para atletas de ultra-resistência seria de 7 a 10 gramas por quilograma de peso ${ }^{15}$.

Brouns et al. (1989) ${ }^{16}$ realizaram uma simulação do Tour de France, em que 13 ciclistas passaram 7 dias em uma câmara respiratória, consumindo uma dieta contendo $60 \%$ de carboidratos; relataram que a oxidação de carboidratos foi maior que a ingestão, indicando depleção de carboidrato endógeno e que a taxa de utilização de carboidratos caiu do dia 4 para o dia 5 , sendo compensada pelo aumento da utilização de gordura.

Os estudos que descrevem o gasto energético e o consumo alimentar durante as competições são poucos, devido às dificuldades metodológicas relativas à coleta de dados. Saris et al. (1989) $)^{17}$, durante o Tour de France $(4.000 \mathrm{~km}$, com montanhas de até $2.700 \mathrm{~m}$ de altitude, durante 22 dias), constataram um consumo médio de $5.881 \mathrm{kcal}$, com máxi- 
mo de $7.714 \mathrm{kcal}$, e um gasto médio de $6.048 \mathrm{kcal}$, com máximo de $7.786 \mathrm{kcal}$, em que o consumo de carboidratos correspondeu a $62 \%$, o de gordura a $23 \%$ e o de proteína a $15 \% ; 49 \%$ do consumo energético foi feito durante as etapas, em que $69 \%$ foram provenientes de carboidratos.

Da mesma forma, Eden e Abernethy (1994) ${ }^{18}$, acompanhando um atleta que percorreu (corrida) $1.005 \mathrm{~km}$ em 9 dias, descreveram um consumo médio de $5.952 \mathrm{kcal}$ por dia, com $62 \%$ provenientes dos carboidratos, $27 \%$ das gordura e $11 \%$ das proteínas.

Gabel et al. (1995) $)^{19}$ relataram um consumo médio de $7.125 \mathrm{kcal}( \pm 340)$ durante um percurso de $3.280 \mathrm{~km}$, em 10 dias, realizado por 2 ciclistas, sendo $63 \%$ da energia consumida provenientes de carboidratos, $27 \%$ de gordura e $10 \%$ de proteína. Case et al. (1995) ${ }^{20}$ observaram um consumo energético similar $( \pm 7.350 \mathrm{kcal})$, mas um percentual de carboidratos maior (71\%), durante uma Ultra-Maratona realizada em clima frio.

Em uma pesquisa realizada no Rio de Janeiro com participantes de uma prova de Triathlon com distâncias Ironman, Ceddia (1993) ${ }^{21}$ encontrou um gasto energético superior aos estudos supracitados $(8.171,1 \mathrm{kcal} \pm 716,7)$ e a ingestão energética média durante a competição menor $(4.175,6 \mathrm{kcal} \pm 248,7)$, em que $94,8 \%$ foram provenientes de carboidratos.

Mertens et al. (1996) 22 acompanharam uma mulher de 43 anos durante uma corrida de $7.250 \mathrm{~km}$, Canadá costa-acosta, em 112 dias $(65 \mathrm{~km} / \mathrm{dia})$, relatando um gasto energético de $5.000 \mathrm{kcal} / \mathrm{dia}$ e um consumo de $3.976 \mathrm{kcal} / \mathrm{dia}$.

Comparando as dietas de treino e pré-competição, Peters e Goetzsche (1997) ${ }^{23}$ acompanharam 150 homens e 23 mulheres que participaram de uma Ultra-Maratona de $90 \mathrm{~km}$; relataram que o consumo energético aumentou nos homens de 2.405 para $3.048 \mathrm{kcal}$ (50 para 57\% de carboidratos) e nas mulheres de 1.786 para $2.167 \mathrm{kcal}(49,5$ para $56,4 \%$ de carboidratos).

Um fato que deve ser considerado em competições que duram vários dias é a dificuldade de se ingerir a quantidade suficiente de carboidratos devido ao curto espaço de tempo entre um estágio e outro da competição e a redução do apetite associada ao esforço excessivo. Dessa forma, torna-se um problema repor os estoques de glicogênio. Outro fato para se considerar é que o grande volume de alimento seja dividido em várias refeições para evitar desconforto causado pela distensão estomacal ${ }^{24}$.

Sendo assim, observa-se que a ingestão alimentar de atletas em competições de longa duração não representa a demanda energética imposta pelo exercício. Esta conduta pode causar efeitos negativos aos competidores, desde a redução do desempenho a problemas mais sérios de saúde.

\section{INTERVENÇÕES NUTRICIONAIS PARA OTIMIZAR O DESEMPENHO}

Frente à grande possibilidade de melhora no desempenho em provas de longa duração que a nutrição pode proporcionar, muitos pesquisadores passaram a estudar recursos nutricionais para melhorar ainda mais o rendimento. Dentre esses recursos, os mais eficazes, tanto antes como durante as competições, são os carboidratos, que estão presentes nas sport bars, nos repositores em gel, nas bebidas energéticas (que também são repositores hidroeletrolíticos) e nas manobras de supercompensação de carboidratos ou sobrecarga glicídica. Outro recurso que vem sendo estudado nos últimos anos é a suplementação de lipídios visando a melhora do desempenho.

\section{CONSUMO E SUPERCOMPENSAÇÃO DE CARBOIDRATOS ANTES DO EXERCÍCIO}

A supercompensação de carboidratos é um modelo clássico de manobra alimentar que objetiva aumentar, chegando a dobrar, os estoques de glicogênio muscular e prolongar significativamente o tempo de exercício até a exaustão ${ }^{5}$. Contudo, esse recurso promove um maior armazenamento de água pelo organismo (para cada 1 grama de carboidrato são armazenadas 3 gramas de água), o que pode causar desconforto em alguns atletas devido ao aumento do peso corporal. No entanto, segundo alguns autores, essa "água extra" pode vir a ser útil na prevenção da desidratação em eventos de endurance $e^{2,5,6,11,14,25,26}$.

Segundo Hawley et al. (1997) ${ }^{27}$, os estoques de glicogênio muscular, em uma pessoa fisicamente ativa que consome uma dieta equilibrada, estão em torno de $125 \mathrm{mmol} / \mathrm{kg}$ de músculo úmido, podendo chegar a mais de $200 \mathrm{mmol} /$ $\mathrm{kg}$ de músculo úmido após um período de supercompensação, sendo que esse aumento melhora o tempo de performance em até $20 \%$ e o desempenho em 2 a $3 \%$, em exercícios que durem mais de 90 minutos.

Para esse aumento de glicogênio ser conseguido, diversos protocolos foram desenvolvidos, como por exemplo o citado por Clark (1997) ${ }^{12}$ e Liebman e Wilkinson (1996) ${ }^{14}$, que sugerem um "afunilamento" do volume e da intensidade do treinamento durante 6 a 15 dias anteriores à competição e, paralelamente, o consumo de carboidratos é progressivamente aumentado para um valor de $70 \%$ ou mais do VET (valor energético total) da dieta durante as últimas 72 horas.

Com relação às refeições pré-competição, recomendase a ingestão de alimentos com baixo índice glicêmico. $\mathrm{O}$ consumo de carboidratos com alto índice glicêmico antes do exercício aumenta a concentração de glicose sanguínea entre 5 e 10 minutos após sua ingestão, o que resulta numa 
maior liberação de insulina pelo pâncreas, causando um declínio na glicose plasmática devido ao rápido transporte deste nutriente para os músculos, o que é conhecido como "hipoglicemia de rebote" 11 . Ao mesmo tempo, a insulina inibe a mobilização de gordura, fato este que pode durar várias horas. Sendo assim, durante o exercício, o carboidrato intramuscular passa a ser catabolizado numa taxa maior que a normal, o que poderia antecipar o aparecimento da fadiga ${ }^{2,11,28}$. No entanto, segundo McArdle et al. (1999) ${ }^{11}$, esse mecanismo ainda não está completamente elucidado e, para minimizar os efeitos da ingestão de carboidratos com alto índice glicêmico antes da atividade, o consumo deve ser feito pelo menos 60 minutos antes, para haver tempo suficiente para o restabelecimento do balanço hormonal antes do início do exercício.

Segundo Ribeiro et al. (1998) ${ }^{29}$, uma refeição pré-competição de baixo índice glicêmico provoca uma menor concentração de glicose e insulina no sangue 30 a 60 minutos após sua ingestão, um maior nível de ácidos graxos livres, uma menor oxidação de carboidratos e um período de realização de 9 a 20 minutos maior durante exercícios de longa duração.

Sendo assim, a refeição pré-competição deve conter de 1 a 5 gramas de carboidrato $/ \mathrm{kg}$ de peso corporal e acontecer entre 1 e 4 horas antes do início da prova ${ }^{5,11,25,26,28-30}$.

Burke et al. (1998) ${ }^{31}$ realizaram um estudo em que 6 ciclistas, em 3 etapas distintas, ingeriram uma refeição préexercício de alto índice glicêmico, uma com baixo índice glicêmico e uma geléia de baixo valor energético e, imediatamente antes e durante o exercício (que durou $\pm 2,5$ horas), consumiram uma bebida com $10 \%$ de glicose. Concluíram os autores que, quando ocorre o consumo de carboidratos durante a atividade, o carboidrato ingerido antes do exercício possui um pequeno efeito, já que não foram encontradas diferenças significativas entre os 3 grupos. Em um outro estudo realizado por Chryssanthopoulos e Williams $(1997)^{32}, 10$ corredores foram divididos em 3 grupos; antes do exercício, 2 grupos consumiram uma solução placebo e 1 grupo consumiu uma refeição com alto teor de carboidratos (88\% proveniente de alimentos com alto índice glicêmico) e durante o exercício os 3 grupos consumiram uma solução com $6,9 \%$ de carboidratos. $\mathrm{O}$ grupo que consumiu a refeição mais a bebida apresentou uma melhora de até $28 \%$ no tempo total de exercício, quando comparado com os outros 2 grupos. $\mathrm{O}$ fato interessante, constatado nos 2 estudos, é que o consumo de alimentos com alto índice glicêmico antes do exercício não causou qualquer tipo de problema, pelo menos quando foram consumidos carboidratos durante o exercício.

Atualmente, concorda-se que a combinação do consumo de carboidratos antes e durante atividades físicas de longa duração promova uma melhora significativa no rendimento $^{28,33}$.

\section{CONSUMO DE CARBOIDRATOS DURANTE O EXERCÍCIO}

Atualmente é amplamente aceito que a ingestão de carboidratos durante os exercícios prolongados irá retardar o aparecimento da fadiga.

Caso não haja consumo de carboidratos durante os exercícios de longa duração, os estoques de glicogênio corporais serão depletados, o que irá provocar uma queda nas concentrações de glicose sanguínea e, como conseqüência, o sistema nervoso central e o metabolismo muscular irão entrar em colapso, provocando a interrupção da atividade $^{2,5,11,26,28,34}$.

Apesar da melhora no desempenho promovida pela suplementação de carboidratos durante a competição, os atletas não devem aumentar o ritmo (intensidade) nos primeiros estágios de eventos de longa duração, o que poderia provocar fadiga antecipadamente. A ingestão de carboidratos permite que um ritmo considerado ideal seja mantido por um grande período de tempo, proporcionando assim um retardo na fadiga ${ }^{5,11,35}$.

Hultman e Greenhaff (1992) ${ }^{34}$ sugerem que o carboidrato seja consumido mais próximo do término da atividade, quando os estoques orgânicos de glicogênio estarão quase que totalmente depletados e, dessa forma, facilitariam a utilização da gordura durante o início do exercício.

Brouns et al. (1989) ${ }^{36}$, em estudo realizado com 13 ciclistas divididos em 2 grupos, ambos permanecendo no interior de uma câmara respiratória durante 7 dias (2 dias de preparação, um dia de descanso, 2 dias pedalando, 1,5 dia de recuperação), relataram que o grupo que recebeu uma bebida à base de maltodextrina $(85 \%)$ junto com a sua dieta normal (60\% de carboidratos) foi capaz de sustentar o balanço energético durante todos os dias, quando comparado com o outro grupo, que recebeu uma solução de $50 \%$ de maltodextrina e $50 \%$ de frutose, e quando comparado com os dados obtidos na primeira parte do estudo, na qual os atletas consumiram apenas a sua dieta normal.

A utilização de bebidas contendo carboidratos é amplamente difundida entre atletas e treinadores. Saris et al. (1989) $)^{17}$ salientaram que, durante o Tour de France, 30\% dos carboidratos consumidos durante as etapas eram provenientes de bebidas e que essa prática parece ser a resposta para manter o balanço energético e de fluidos em condições extremas de exercício.

Recomenda-se que a concentração de carboidratos fique entre $4 \%$ e $8 \%$, devendo-se dar preferência para soluções de polímeros de glicose, que facilitam o esvaziamento gástrico ${ }^{5,11,12,14,24-26,28,29,34,35,37,38}$. 
Singh et al. (1994) ${ }^{30}$ recomendam que durante exercícios prolongados sejam consumidos de 250 a $300 \mathrm{ml}$ de uma bebida com concentração de 6 a $8 \%$ de carboidratos e eletrólitos a cada 30 minutos.

Dennis et al. (1997) ${ }^{39}$ estimam que aproximadamente 20 gramas de carboidrato consumido são oxidados durante a primeira hora de exercício; sendo assim, orientam para que os atletas devam consumir cerca de $100 \mathrm{ml}$ de uma bebida contendo de 3 a $5 \%$ de carboidratos, para depois aumentarem essa concentração para cerca de $10 \%$ de carboidratos, a fim de igualar a taxa de oxidação da glicose sanguínea (aproximadamente 1 grama por minuto).

Porém, Costill e Wilmore (1999) ${ }^{5}$ ressaltam que até hoje nenhuma bebida considerada ideal foi desenvolvida, levando-se em consideração as diferentes fases do exercício e a composição de carboidratos e eletrólitos presente no repositor. Além disso, Clark (1998) ${ }^{12}$ sinaliza para que o atleta experimente bebidas diferentes durante os treinamentos para descobrir com qual ele melhor se adapta.

Um outro recurso muito utilizado pelos atletas de ultraresistência durante as competições são os repositores em gel ou barra (sports bars). A concentração de carboidratos nestes repositores varia entre 40 e $80 \%$, sendo compostos basicamente por maltodextrina, glicose, sacarose e frutose, o que fornece grande quantidade de energia rapidamente disponível. Além disso, esse tipo de repositor é encontrado nos mais diversos sabores, de frutas a chocolate, o que agrada a maioria dos atletas ${ }^{40}$.

Rauch et al. (1999) ${ }^{41}$, em estudo realizado com 6 ciclistas, concluíram que o consumo de uma sports bar contendo uma mistura de $7 \mathrm{~g}$ de gordura, $14 \mathrm{~g}$ de proteína e $19 \mathrm{~g}$ de carboidrato, quando comparada com uma quantidade equicalórica de carboidratos, aumentou a taxa de oxidação de gorduras significativamente em exercício submáximo com duração de 5,5 horas. Porém, 2 ciclistas não completaram uma etapa subseqüente de exercício de alta intensidade quando consumiram a barra, enquanto que todos que consumiram apenas o carboidrato completaram todas as etapas.

Porém, Clark (1998) ${ }^{12}$ cita que os atletas precisam estar completamente adaptados a esse tipo de repositor, já que a alta concentração e o tipo de carboidrato que eles possuem, o sabor e os demais nutrientes neles contidos podem vir a causar problemas gástricos e intestinais durante a competição.

\section{SUPLEMENTAÇÃO COM LIPÍDIOS}

Os carboidratos são o substrato energético para atividades aeróbias de longa duração, porém as reservas corporais de glicogênio são limitadas e podem ser totalmente depletadas em eventos atléticos desta natureza. Sendo assim, pode ser vantajoso otimizar a utilização do lipídio (ácidos graxos livres) como fonte de energia, poupando os estoques de glicogênio para os estágios finais da competição.

Vem sendo sugerido que a capacidade de sustentar o exercício pode ser prolongada se juntamente com os estoques de glicogênio a oferta de lipídios for aumentada imediatamente antes do exercício e, como a taxa de oxidação dos ácidos graxos livres é dependente da concentração sanguínea dos mesmos, algumas técnicas foram desenvolvidas para promover esse aumento: dietas pobres em carboidratos e ricas em lipídios, infusão de emulsões de triglicerídeos e a ingestão de triglicerídeos de cadeia média (TCM $)^{3,}$ $11,26,34$.

Estudos demonstram que os TCM podem ser uma importante fonte de energia exógena quando combinados com carboidratos. No entanto, a suplementação de TCM combinados com carboidratos ( $\mathrm{CHO}$ ) ainda não demonstrou vantagem, durante o exercício de longa duração, que justifique uma mudança na composição da dieta ${ }^{11,26,42,43}$.

Sobre isso, Jeukendrup et al. (1996) ${ }^{44}$, com o objetivo de verificar a taxa de oxidação de TCM, avaliaram 8 atletas de elite, durante quatro sessões de 90min de exercício em bicicleta ergométrica ( $57 \%$ vंO máx). Os atletas consumiram duas soluções, antes e durante o exercício, uma contendo somente $\mathrm{CHO}(15 \%)$ e outra CHO + TCM. Embora a oxidação total de lipídios tenha aumentado marcadamente, a oxidação de TCM aumentou marginalmente. A contribuição de TCM ao dispêndio energético total foi pequena, em torno de 6-8\%.

Os TCM não tendem a ser estocados como gordura corporal devido à sua facilidade de oxidação. Os TCM contêm ácidos graxos saturados com 8 a 10 átomos de carbono. Durante a digestão, os TCM são hidrolisados pela ação da lipase na boca, estômago e intestino em glicerol e ácidos graxos de cadeia média (AGCM). A solubilidade em água dos AGCM permite que eles sejam rapidamente transportados através da mucosa intestinal diretamente para a corrente sanguínea (veia porta). Uma vez nos tecidos, os AGCM são imediatamente transportados através da membrana plasmática, onde eles difundem através da membrana interna da mitocôndria para oxidação, independentemente do sistema carnitina-acetil-CoA transferase. Sendo assim, especula-se que a suplementação com esse tipo de lipídio possa poupar o glicogênio muscular e hepático no decorrer de exercícios aeróbios de alta intensidade ${ }^{11,45}$.

Em teoria, a ingestão de TCM pode aumentar os níveis plasmáticos de ácidos graxos. No entanto, atletas bem treinados que ingeriram $\mathrm{CHO}+\mathrm{TCM}$ ou $\mathrm{CHO}$ não apresentaram diferença significativa na quebra de glicogênio entre as 
sessões ou no quociente respiratório (R) durante o exercício. Isto pode ser atribuído à pequena quantidade de TCM $(30 \mathrm{~g})$ tolerada pelo trato gastrintestinal ${ }^{46}$. Van Zyl et al. $(1996)^{47}$ sugeriram que a quantidade de TCM foi pequena para exercer efeitos positivos no rendimento. Eles suplementaram os atletas com $86 \mathrm{~g}$ de TCM durante 2 horas de exercício seguido por um sessão de $40 \mathrm{~km}$, quando observaram uma redução nos estoques de glicogênio e uma melhora no rendimento com a solução de CHO + TCM. Os autores não relataram nenhum desconforto gastrintestinal por parte dos atletas. Contudo, Jeukendrup et al. $(1998)^{48}$, em um experimento similar oferecendo $86 \mathrm{~g}$ de TCM em solução de TCM e TCM + CHO, não encontraram resultados positivos no rendimento atlético. No entanto, quando apenas TCM foi ingerido observou-se um declínio na performance relacionado ao desconforto gastrintestinal relatado pelos atletas.

Recentemente, Angus et al. $(2000)^{49}$, verificando o efeito da ingestão de $\mathrm{CHO}$ e $\mathrm{CHO}+\mathrm{TCM}$ no metabolismo e no rendimento, avaliaram 8 atletas de endurance percorrendo $100 \mathrm{~km}$ o mais rápido possível, em bicicleta ergométrica. As soluções eram compostas por $\mathrm{CHO} 6 \%$ ou $\mathrm{CHO} 6 \%+$ TCM $4,2 \%$ ou placebo, consumidas a cada $15 \mathrm{~min}(250 \mathrm{ml})$. Os resultados demonstraram que a ingestão de carboidrato durante o exercício aumentou o rendimento, comparada com a solução placebo, mas a adição de TCM não resultou em nenhum aumento de performance.

Além da suplementação com TCM, as dietas ricas em lipídios vêm despertando interesse como uma ferramenta auxiliar no aumento do rendimento em provas de enduran$c e$, visto que, teoricamente, dietas hiperlipídicas promovem um aumento na capacidade de oxidação de ácidos graxos livres pelo organismo ${ }^{43}$.

Segundo Hultman e Greenhaff $(1992)^{34}$, mesmo quando os estoques de glicogênio não estão reduzidos, o consumo de uma refeição rica em lipídios antes do exercício vai reduzir a utilização de carboidratos e aumentar a oxidação dos ácidos graxos livres, até mesmo em intensidades em que o glicogênio muscular é a principal fonte de energia. No entanto, Brooks et al. (1996) ${ }^{25}$ citam que, apesar dos lipídios serem uma importante fonte de energia durante exercícios prolongados, os atletas não devem consumir quantidades maiores de lipídios na dieta.

Lambert et al. (1997) ${ }^{50}$ sugerem que uma adaptação dietética à base de lipídios (refeições com mais de $70 \%$ de lipídios), durante 2 a 4 semanas antes de eventos de ultraresistência com intensidade de baixa a moderada $(<70 \%$ V̇O2máx), poderia melhorar o desempenho. Os autores ressaltam que os efeitos da suplementação de lipídios ainda não foram estudados em atividades com intensidades mais elevadas. Da mesma forma, Hawley et al. $(1998)^{51}$ citam que em atletas de resistência treinados a utilização do substrato energético durante exercício submáximo $(60 \%$ vंO2máx) pode ser significativamente alterada pela ingestão de um dieta rica em lipídios e pobre em carboidrato (60-70\% e 15-20\%, respectivamente) por um período de 7 a 10 dias. Entretanto, esse tipo de adaptação parece não ter efeito sobre a taxa de utilização do glicogênio muscular e do rendimento em exercícios prolongados com intensidade moderada.

Em estudo interessante desenvolvido por Starling et al. $(1997)^{52}$, os indivíduos ingeriram uma dieta com alto teor de carboidratos (83\%) ou com alto teor de lipídios (68\%) durante 12 horas após uma sessão de exercícios em bicicleta ergométrica a $65 \%$ vंO2máx por $120 \mathrm{~min}$. Após 12 horas de jejum, foi realizada biópsia muscular e uma sessão de exercício correspondente a 1.600kj. A dieta rica em lipídios aumentou em $21 \%$ a concentração intramuscular de triglicerídeos, ao contrário da dieta rica em carboidratos. Em paralelo a estes resultados, a concentração de glicogênio muscular após a dieta rica em carboidratos foi maior, resultando em melhor rendimento no exercício de 1.600kj, quando comparado à dieta rica em lipídios.

Jeukendrup et al. (1998) $)^{42}$ recomendam cautela na prescrição de dietas hiperlipídicas, uma vez que existem poucas evidências sobre os efeitos desta intervenção no rendimento de atletas de endurance.

Sendo assim, observa-se que não existem evidências científicas suficientes que justifiquem a suplementação de TCM durante o exercício ou o consumo de dietas ricas em lipídios nas semanas que antecedem um evento de ultra-resistência.

\section{CONCLUSÃO}

Vem sendo demonstrado, ao longo dos últimos anos, que a manipulação dietética pode ser empregada como fator otimizante do desempenho de atletas de ultra-resistência.

Os atletas ou praticantes de provas de longa duração precisam de uma alimentação adequada às necessidades energéticas da modalidade esportiva da qual participam.

Atletas de ultra-resistência devem consumir aproximadamente $70 \%$ ou mais, ou de 7 a 10 gramas por quilograma de peso corporal de carboidratos em suas dietas diárias, podendo vir a aumentar essa percentagem dias antes da prova para promover um aumento nas reservas corporais de glicogênio. $\mathrm{O}$ consumo de carboidratos durante a competição deve ser encorajado, principalmente através de soluções contendo esse nutriente, pois além de fornecer energia irá repor fluidos e eletrólitos perdidos durante o esforço. Um volume de $250 \mathrm{ml}$ de uma solução contendo de 4 a $8 \%$ de carboidratos a cada 30 minutos parece ser o 
ideal. Os carboidratos em forma de gel ou barras são outra forma muito utilizada pelos atletas, porém, a adaptação a esse tipo de repositor deve ser testada, pois tanto a concentração de carboidratos como o sabor podem vir a causar problemas gástricos.

A utilização de lipídios como suplemento ainda precisa de mais estudos que confirmem a sua eficácia. Esses e alguns outros aspectos nutricionais relacionados com exercícios de ultra-resistência merecem maiores e mais pro-

\section{REFERÊNCIAS}

1. Moreira SB. Equacionando o treinamento: A matemática das provas longas. Rio de Janeiro: Shape, 1996.

2. Miller GD. Carboidratos na ultra-resistência e no desempenho atlético. In: Wolinsky I, Hickson JF Jr, editores. Nutrição no exercício e no esporte. $2^{\underline{a}}$ ed. São Paulo: Roca, 1996:51-67.

3. McMurray RG, Anderson JJB. Introdução à nutrição no exercício e no esporte. In: Wolinsky I, Hickson JF Jr, editores. Nutrição no exercício e no esporte. $2^{\underline{a}}$ ed. São Paulo: Roca, 1996:1-14.

4. Garcia-Rovés PM, Terrados N, Fernández SF, Patterson AM. Macronutrients intake of top level cyclists during continuous competition - change in the feeding pattern. Int J Sports Med 1998;19:61-7.

5. Costill DL, Wilmore JH. Physiology of sport and exercise. $2^{\text {nd }}$ ed. Champaing: Human Kinetics, 1999.

6. Basset DR Jr, Nagle FJ. Metabolismo energético no treino e exercício. In: Wolinsky I, Hickson JF Jr, editores. Nutrição no exercício e no esporte. $2^{2}$ ed. São Paulo: Roca, 1996:149-69.

7. Stroud M. The nutritional demands of very prolonged exercise in man. Proc Nutr Soc 1998;57:1:55-61.

8. Fox EL, Bowers RW, Foss ML. The physiological basis for exercise and sports. $5^{\text {rd }}$ ed. USA: Brown \& Benchmark, 1993.

9. Grandjean AC, Ruud JS. Nutrition for cyclists. Clin Sports Med 1994; 13:1:235-47.

10. Katch FL, Mcardle WD. Nutrição, saúde e exercício. $4^{\underline{a}}$ ed. Rio de Janeiro: Medsi, 1996.

11. McArdle WD, Katch FI, Katch VL. Sports \& exercise nutrition. USA: Lippincott, Williams \& Wilkins, 1999.

12. Clark N. Guia de nutrição desportiva. $2^{\mathfrak{a}}$ ed. Porto Alegre: Artmed, 1998.

13. Scott D. Dave Scott's triathlon training. New York: Simon \& Schuster, 1986.

14. Liebman M, Wilkinson JG. Metabolismo de carboidratos e condicionamento físico. In: Wolinsky I, Hickson JF Jr, editores. Nutrição no exercício e no esporte. $2^{\underline{a}}$ ed. São Paulo: Roca, 1996:15-50.

15. Maughan RJ, editor. Nutrition in sport. IOC Medical Commission, Blackwell Science, 2000.

16. Brouns F, Saris WH, Stroecken J, Beckers E, Thijssen R, Rehrer NJ, et al. Eating, drinking and cycling. A controlled Tour de France simulation study, part I. Int J Sports Med 1989;10(Suppl 1):S32-40.

17. Saris WH, Van Erp-Baart MA, Brouns F, Westerterp KR, Ten Hoor F. Study on food intake and energy expenditure during extreme sustained exercise: The Tour de France. Int J Sports Med 1989;10(Suppl 1):S2631.

18. Eden BD, Abernethy PJ. Nutritional intake during an ultraendurance running race. Int J Sport Nutr 1994;4:2:166-74.

19. Gabel KA, Aldous A, Edgington C. Dietary intake of two elite male cyclists during 10-day, 2.050-mile ride. Int J Sport Nutr 1995;5:1:56-61. fundas investigações; com o aumento da popularidade desse tipo de competições, esse é um campo que tende a crescer ainda mais.

Sendo assim, atletas de ultra-resistência devem sempre seguir as orientações do(a) seu(sua) técnico(a), que deve trabalhar diretamente em conjunto com um(a) nutricionista, e ambos precisam estar sempre bem atualizados, para que a melhora do desempenho nas competições possa ser alcançada com sucesso e de forma saudável.

20. Case S, Evans D, Tibbets G, Miller D. Dietary intakes of participants in the Iditasport Human Powered Ultra-Marathon. Alaska Medicine 1995; 37:1:20-4.

21. Ceddia RB. Perfil da perda hídrica e da ingestão de nutrientes durante o exercício e seus efeitos sobre a performance de atletas participantes de uma competição de Ironman Triathlon. Tese de Mestrado, Escola de Educação Física e Desportos, Universidade Federal do Rio de Janeiro, 1993

22. Mertens DJ, Rhind S, Berkhoff F, Dugmore D, Shek PN, Shephard RJ Nutritional, immunologic and psychological responses to a 7,250 km run. J Sports Med Phys Fitness 1996;36:2:132-8.

23. Peters EM, Goetzsche JM. Dietary practices of South African ultradistance runners. Int J Sport Nutr 1997;7:2:80-103.

24. Nielsen B. Diet, vitamins and fluids: Intake before and after prolonged exercise. In: Shephard RJ, Astrand P-O, editores. Endurance in sport. International Olympic Committee, 1992:297-311.

25. Brooks GA, Fahey TD, White TP. Exercise physiology: Human bioenergetics and its applications. $2^{\text {nd }}$ ed. California: Mayfield Publishing Company, 1996.

26. Williams MH. The ergogenics edge. Champaign: Human Kinetics, 1998.

27. Hawley JA, Schabort EJ, Noakes TD, Dennis SC. Carbohydrate-loading and exercise performance. An update. Sports Med 1997;24:2:73-81.

28. Powers SK, Howley ET. Exercise physiology: Theory and its application to fitness and performance. $3^{\text {rd }}$ ed. USA: Brown \& Benchmark, 1997.

29. Ribeiro BG, Pierucci APTR, Soares EA, Carmo MGT. A influência dos carboidratos no desempenho físico. Rev Bras Med Esporte 1998;4:6: 197-202.

30. Singh A, Pelletier PA, Deuster PA. Dietary requirements for ultra-endurance exercise. Sports Med 1994;18:5:301-8.

31. Burke LM, Claassen A, Hawley JA, Noakes TD. Carbohydrate intake during prolonged cycling minimizes effect of glycemic index of preexercise meal. J Appl Physiol 1998;85:6:2220-6.

32. Chryssanthopoulos C, Williams C. Pre-exercise carbohydrate meal and endurance running capacity when carbohydrates are ingested during exercise. Int J Sports Med 1997;18:7:543-8.

33. Rauch HG, Hawley JA, Noakes TD, Dennis SC. Fuel metabolism during ultra-endurance exercise. Pflugers Arch 1998;436:2:211-9.

34. Hultman E, Greenhaff PL. Food stores and energy reserves. In: Shephard RJ, Astrand, P-O, editores. Endurance in sport. International Olympic Committee, 1992:127-35.

35. Williams C. Dietary macro- and micronutrient requirements of endurance athletes. Proc Nutr Soc 1998;57:1:1-8.

36. Brouns F, Saris WH, Stroecken J, Beckers E, Thijssen R Rehrer NJ, et al. Eating, drinking and cycling. A controlled Tour de France simulation study, part II. Effect of diet manipulation. Int J Sports Med 1989;10 (Suppl 1):S41-8. 
37. Puhl SM, Buskirk ER. Bebidas nutrientes para o exercício e o esporte. In: Wolinsky I, Hickson JF Jr, editores. Nutrição no exercício e no esporte. $2^{\underline{a}}$ ed. São Paulo: Roca, 1996:285-320.

38. Tsintzas K, Williams C. Human muscle glycogen metabolism during exercise. Effect of carbohydrate supplementation. Sports Med 1998;25: $1: 7-23$.

39. Dennis SC, Noakes TD, Hawley JA. Nutritional strategies to minimize fatigue during prolonged exercise: Fluid, electrolyte and energy replacement. J Sports Sci 1997;15:3:305-13.

40. Pierucci APTR, Casé FB, Sato PHR, Gomes AJS, Ribeiro BG, Carvalho LMJ, et al. Elaboration of a high carbohydrate supplement for endurance athletes. Alimentaria 2000;318:81-9.

41. Rauch HG, Hawley JA, Woodey M, Noakes TD, Dennis SC. Effects of ingesting a sports bar versus glucose polymer on substrate utilization and ultra-endurance performance. Int J Sports Med 1999;20:4:252-7.

42. Jeukendrup AE, Saris WHM, Wagenmakers AJM. Fat metabolism during exercise: A review. Int J Sports Med 1998;19:371-9.

43. Brouns F, van der Vusse GJ. Utilization of lipids during exercise in human subjects: metabolic and dietary constraints. Br J Nutr 1998;79:11728.

44. Jeukendrup AE, Saris WH, Van Diesen R, Brouns F, Wagenmakers AJM. Effect of endogenous carbohydrate availability on oral medium-chain triglyceride oxidation during prolonged exercise. J Appl Physiol 1996; 80:949-54.
45. Berning JR. The role of medium-chain triglyceride in exercise. Int $\mathrm{J}$ Sport Nutr 1996;6:121-33.

46. Jeukendrup AE, Saris WH, Brouns F, Halliday D, Wagenmakers JM. Effects of carbohydrate (CHO) and fat supplementation on CHO metabolism during prolonged exercise. Metabolism 1996;45:915-21.

47. Van Zyl CG, Lambert EV, Hawley JA, Noakes TD, Dennis SC. Effects of medium-chain triglyceride ingestion on fuel metabolism and cycling performance. J Appl Physiol 1996;80:2217-25.

48. Jeukendrup AE, Thielen JJ, Wagenmakers AJ, Brouns F, Saris WH. Effect of medium-chain triacylglycerol and carbohydrate ingestion during exercise on substrate utilization and subsequent cycling performance. Am J Clin Nutr 1998;67:397-404.

49. Angus DJ, Hargreaves M, Dancey J, Febbraio MA. Effect of carbohydrate or carbohydrate plus medium-chain triglyceride ingestion on cycling time trial performance. J Appl Physiol 2000;88:113-9.

50. Lambert EV, Hawley JA, Goedecke J, Noakes TD, Dennis SC. Nutritional strategies for promoting fat utilization and delaying the onset of fatigue during prolonged exercise. J Sports Sci 1997;15:3:315-24.

51. Hawley JA, Brouns F, Jeukendrup A. Strategies to enhance fat utilization during exercise. Sports Med 1998;25:4:241-57.

52. Starling RD, Trappe TA, Parcell AC, Kerr CG, Fink WJ, Costill DL. Effects of diet on muscle triglyceride and endurance performance. J Appl Physiol 1997;82:1185-9. 